\title{
An optimized photoelectron track reconstruction method for photoelectric $\mathrm{X}$-ray polarimeters
}

\author{
Takao Kitaguchi ${ }^{\text {a,b,*, Kevin Black }}{ }^{\text {c,d }}$, Teruaki Enoto ${ }^{\mathrm{e}}$, Yasushi Fukazawa ${ }^{\text {a,b,f }}$, Asami Hayato ${ }^{g}$, \\ Joanne E. Hill ${ }^{c}$, Wataru B. Iwakiri ${ }^{\text {h}}$, Keith Jahoda ${ }^{c}$, Philip Kaaret ${ }^{i}$, Ross McCurdy ${ }^{i}$, \\ Tsunefumi Mizuno ${ }^{\mathrm{a}, \mathrm{b}, \mathrm{f}}$, Toshio Nakano ${ }^{\mathrm{g}}$, Toru Tamagawa ${ }^{\mathrm{g}}$ \\ a Department of Physical Science, Hiroshima University, 1-3-1 Kagamiyama, Higashi-Hiroshima, Hiroshima, 739-8526, Japan \\ b Core Research for Energetic Universe, Hiroshima University, 1-3-1, Kagamiyama, Higashi-Hiroshima, Hiroshima, 739-8526, Japan \\ c NASA Goddard Space Flight Center, Greenbelt, MD 20771, USA \\ ${ }^{d}$ Rock Creek Scientific, 1400 East-West Hwy, Silver Spring, MD, 20910, USA \\ e The Hakubi Center for Advanced Research, Kyoto University, Kyoto, 606-8302, Japan \\ ${ }^{\mathrm{f}}$ Hiroshima Astrophysical Science Center, Hiroshima University, 1-3-1 Kagamiyama, Higashi-Hiroshima, Hiroshima, 739-8526, Japan \\ ${ }^{8}$ RIKEN Nishina Center, 2-1 Hirosawa, Wako, Saitama, 351-0198, Japan \\ ${ }^{\text {h }}$ RIKEN MAXI team, 2-1 Hirosawa, Wako, Saitama, 351-0198, Japan \\ ${ }^{\text {i }}$ University of Iowa, Iowa City, IA, 52242, USA
}

\section{A $\quad$ R}

\section{Keywords:}

X-ray

Polarimetry

Photoelectric polarimeter

Time projection chamber

Tracking algorithm

\begin{abstract}
A $\quad$ B $\quad S \quad T \quad R \quad A \quad C \quad T$
We present a data processing algorithm for angular reconstruction and event selection applied to 2-D photoelectron track images from X-ray polarimeters. The method reconstructs the initial emission angle of a photoelectron from the initial portion of the track, which is obtained by continuously cutting a track until the image moments or number of pixels fall below tunable thresholds. In addition, event selection which rejects round tracks quantified with eccentricity and circularity is performed so that polarimetry sensitivity considering a trade-off between the modulation factor and signal acceptance is maximized. The modulation factors with applying track selection are $26.6 \pm 0.4,46.1 \pm 0.4,62.3 \pm 0.4$, and $61.8 \pm 0.3 \%$ at $2.7,4.5,6.4$, and $8.0 \mathrm{keV}$, respectively, using the same data previously analyzed by Iwakiri et al. (2016), where the corresponding numbers are $26.9 \pm 0.4,43.4 \pm 0.4,54.4 \pm 0.3$, and $59.1 \pm 0.3 \%$. The method improves polarimeter sensitivity by $5 \%-10 \%$ at the high energy end of the band previously presented (Iwakiri et al. 2016).
\end{abstract}

(C) 2017 Elsevier B.V. All rights reserved.

\section{Introduction}

Recent progress in micropattern gas detectors enables us to sensitively track charged particles with energies down to $1 \mathrm{keV}$. The angular distribution of photoelectrons is sensitive to the electric field vector (or polarization direction) of incident photons. Since the photoelectric effect is the dominant interaction of X-rays, the micropattern gas polarimeter is expected to open up a new window in cosmic X-ray polarimetry.

The angular distribution, $d \sigma / d \Omega$, of the K-shell photoelectron emission in the non-relativistic region is theoretically given by:

$\frac{d \sigma}{d \Omega} \propto \frac{\sin ^{2} \theta}{(1-\beta \cos \theta)^{4}} \cos ^{2} \phi$,

where $\beta$ is the ratio of the photoelectron velocity to the speed of light, $\phi$ is the azimuth angle of the X-ray electric vector, and $\theta$ is the polar angle with respect to the incident X-ray direction (e.g. Ref. [1]). Since the angular distribution is represented by the product of independent functions of $\theta$ and $\phi$, a 2-D photoelectron track projected onto a plane perpendicular to the incident X-ray direction is sufficient to measure the X-ray polarization direction. The projected distribution is given by $\cos ^{2} \phi$, meaning that the modulation factor, defined as the ratio of sinusoidal amplitude to unmodulated offset, is intrinsically $100 \%$ for all $\theta$ in the photoelectric effect. ${ }^{1}$

Two major types of micropattern gas polarimeters have been developed to image the photoelectron track with sufficient resolution to

\footnotetext{
1 This is a simpler case than for a Compton scattering polarimeter where the intrinsic modulation factor approaches zero for forward and backward scattering; this situation requires 3-D tracking of the scattered X-rays to achieve the maximum polarization sensitivity (e.g. Refs. [2,3])
}

\footnotetext{
* Corresponding author at: Department of Physical Science, Hiroshima University, 1-3-1 Kagamiyama, Higashi-Hiroshima, Hiroshima, 739-8526, Japan. E-mail address: kitaguti@astro.hiroshima-u.ac.jp (T. Kitaguchi).
} 
determine the emission angle: a position-sensitive detector with a 2-D readout system called the gas pixel detector (GPD) [4,5] and a positioninsensitive but more efficient polarimeter with 1-D strip electrodes using the time projection chamber (TPC) technique $[6,7]$. In the GPD, the photoelectron track drifts parallel to the original direction of the photon, while in the TPC the drift is perpendicular. This difference enables imaging of astronomical sources in the GPD and higher efficiency in the TPC. Despite this fundamental difference, both the GPD and TPC produce 2-D tracks. In both polarimeters, an algorithm to reconstruct the initial angle of photoelectrons derived from a 2-D track image is essential to maximize polarimetry sensitivity (e.g. Ref. [6] for the TPC and Refs. [8,9] for the GPD). The new algorithm described here extends and improves our previous method in Ref. [6]. In addition, it adopts measured data-driven approach, while the method in Ref. [8] needs helps with a Monte Carlo simulation to know the real interaction position and bring a reconstructed position close to it.

This paper describes the angular reconstruction and track selection for 2-D images with image moments and its verification using experimental data from the TPC polarimeter we have developed [7]. The method is applicable to the other photoelectric polarimeters, such as the GPD polarimeter with hexagonal pixels. Section 2 briefly reviews the polarimeter and the experimental setup. We describe the angular reconstruction in Section 3 and the track selection in Section 4, and lastly, conclude the study in Section 5. Throughout this paper, all errors are given at the $1 \sigma$ confidence level unless otherwise stated.

\section{Experimental setup and track images}

The TPC polarimeter we have developed for measuring linear polarization of cosmic X-rays is capable of taking a $3.6-\mathrm{mm}$ square image divided into 30-by-30 pixels to track a photoelectron induced by an incident X-ray. Pure dimethyl ether (DME) is sealed as target gas at a pressure of 190 Torr in the polarimeter chamber. The polarimeter was tested with linearly polarized X-rays at the X-19A beamline at the National Synchrotron Light Source facility in Brookhaven National Laboratory. The details of the measurement setup and data processing are available in Ref. [10]. Data sets at 10 monochromatic energies (2.7$8.0 \mathrm{keV}$ ) were used in this work, each having approximately 50,000 events. In addition, the polarimeter was irradiated with the X-ray beam at three different positions, which correspond to the electron drift length from the interaction point to the nearest readout electrode of $0.6,0.8$, and $1.0 \mathrm{~cm}$, where the position of the $0.8 \mathrm{~cm}$ drift length is designed to be at the optical axis of the X-ray mirror. The three data sets at each energy are combined into a single data set and analyzed in the same manner because the drift length would be unknown in a measurement of cosmic X-rays. The beam polarization direction was aligned diagonally with the square image. The beam polarization was separately measured with a scattering polarimeter and was determined to be $94 \%$ [11].

Typical track images taken with $2.7,4.5,6.4$, and $8.0 \mathrm{keV}$ X-rays are shown in Fig. 1. Pixels in which the measured signal is less than three times the noise are set to zero. The electron continuous-slowingdown approximation ranges at 2.2, 4.0, 5.9, and $7.5 \mathrm{keV}$, which are the photoelectron energies calculated by subtracting the K-shell binding energy of oxygen in DME from the incident X-ray energies, are estimated to be $0.31,0.90,1.8$, and $2.7 \mathrm{~mm}$, (or $2.6,7.4,15$, and 22 pixel), respectively. They were calculated according to the analytic formula given in Ref. [12], although it was validated for electrons in condensed materials. For the lowest energies, and shortest tracks, the images are dominated by electron diffusion. The standard deviation is estimated to be $0.15 \mathrm{~mm}$ or 1.3 pixel for $0.8 \mathrm{~cm}$ drift distance with Magboltz [13]. Above $4.5 \mathrm{keV}$, tracks are clearly elongated. The high charge density region corresponds to the Bragg peak at the end of the track. This is because the electron ionization loss per unit length depends on the inverse of its energy according to an approximation of the Bethe formula in the low-energy limit: $-d E / d x \propto E^{-1}$, and therefore the charge distribution tends to be biased along its trajectory. In addition, in the higher energy range, some of the tracks are curved due to large-angle scattering of the photoelectron with a gas molecule.

\section{Angular reconstruction}

An accurate and robust method to reconstruct the initial ejection angle of a photoelectron from the various track images (see Fig. 1) is vital to achieve high polarization sensitivity. In digital image processing, image orientation, $\Phi$, having the centroid as its pivot is given by:

$\Phi=\frac{1}{2} \arctan \left(\frac{2 \mu_{11}}{\mu_{20}-\mu_{02}}\right)$

in general (e.g. Ref. [14]). In the above equation, $\mu_{i j}$ is a centralized $(i, j)$-moment of a 2-D image in which the pixel at $(x, y)$ has a charge amount of $Q_{x y}$ :

$\mu_{i j}=\sum_{x} \sum_{y}(x-\bar{x})^{i}(y-\bar{y})^{j} Q_{x y}$,

where $(\bar{x}, \bar{y})$ is the charge centroid position. The $\Phi$ direction is the same as the major principal axis of the charge distribution. Although $\Phi$ calculated with the atan 2 function ranges from $-\pi / 2$ to $\pi / 2$, it is expandable to all angles $[-\pi, \pi]$ by using the sign of the third moment or skewness, which is sensitive to bias of the charge distribution, along the major principal axis with respect to the centroid. Fig. 2(a) illustrates an example of the above track reconstruction hereafter called "the singlestage reconstruction". It is clear that the single-stage method fails to accurately reconstruct the initial photoelectron angle for curved tracks which often appear in the higher energy range.

Since the track direction is randomized by Coulomb scattering with gas molecules, the charge distribution near the beginning of the track carries the most information about the initial direction and thus the photon electric field. In order to improve the estimated track direction for curved tracks, the image region with the track end should be disregarded. In the previous works [6,11], we divided the track image by the minor principal axis, which is perpendicular to the major axis with respect to the centroid, ignore the half with the track end determined with the sign of the third moment along the major axis, and calculate the angle with the remaining half (which includes the interaction point) from Eq. (2). In the cutting of pixels with their position, a charge amount in each pixel is assumed to be located at its center. This revised method, hereafter called "the two-stage reconstruction" and illustrated in Fig. 2(b), is applied to elongated tracks (eccentricity $e>0.8$ ), while the single-stage method is applied to tracks with $e \leq 0.8$. (Eccentricity is defined in Section 4.) Further improvement is possible for some tracks; the improved angle in Fig. 2(b) is closer to the initial direction, but does not yet account for all of the visible curvature in the first half of the track.

In order to adaptively remove the curved part of a track image and keep the beginning without overcutting, we use image moments up to the second order for not only a grayscale image with $Q_{x y}$ but also its binary image created by image thresholding with the same value (three times the noise) as that described in Section 2. We hereafter distinguish the two moments by defining $\mu_{i j}$ and $\mu_{i j}^{\prime}$ for grayscale and binary images, respectively. For example, the zeroth moment for a binary track image is $\mu_{00}^{\prime}$ which represents the number of hit pixels. We can calculate the maximum and minimum of second moments, $M_{2}^{\max }$ and $M_{2}^{\min }$, which corresponds to the standard deviations along the major and minor axes, respectively, as follows:

$$
\begin{aligned}
& M_{2}^{\max }=\frac{1}{2}\left[\mu_{20}+\mu_{02}+\sqrt{\left(\mu_{20}-\mu_{02}\right)^{2}+4 \mu_{11}^{2}}\right], \\
& M_{2}^{\min }=\frac{1}{2}\left[\mu_{20}+\mu_{02}-\sqrt{\left(\mu_{20}-\mu_{02}\right)^{2}+4 \mu_{11}^{2}}\right] .
\end{aligned}
$$

The track image is repeatedly cut off in half pixel steps along the major axis of the entire image until the remaining number of pixels or the maximum second moment falls below set thresholds. The modulation factor derived from all the data is the highest when the threshold condition is set to be

$\left(\mu_{00}^{\prime} \leq 23\right)$ or $\left(M_{2}^{\max } \leq 3.0\right)$, 


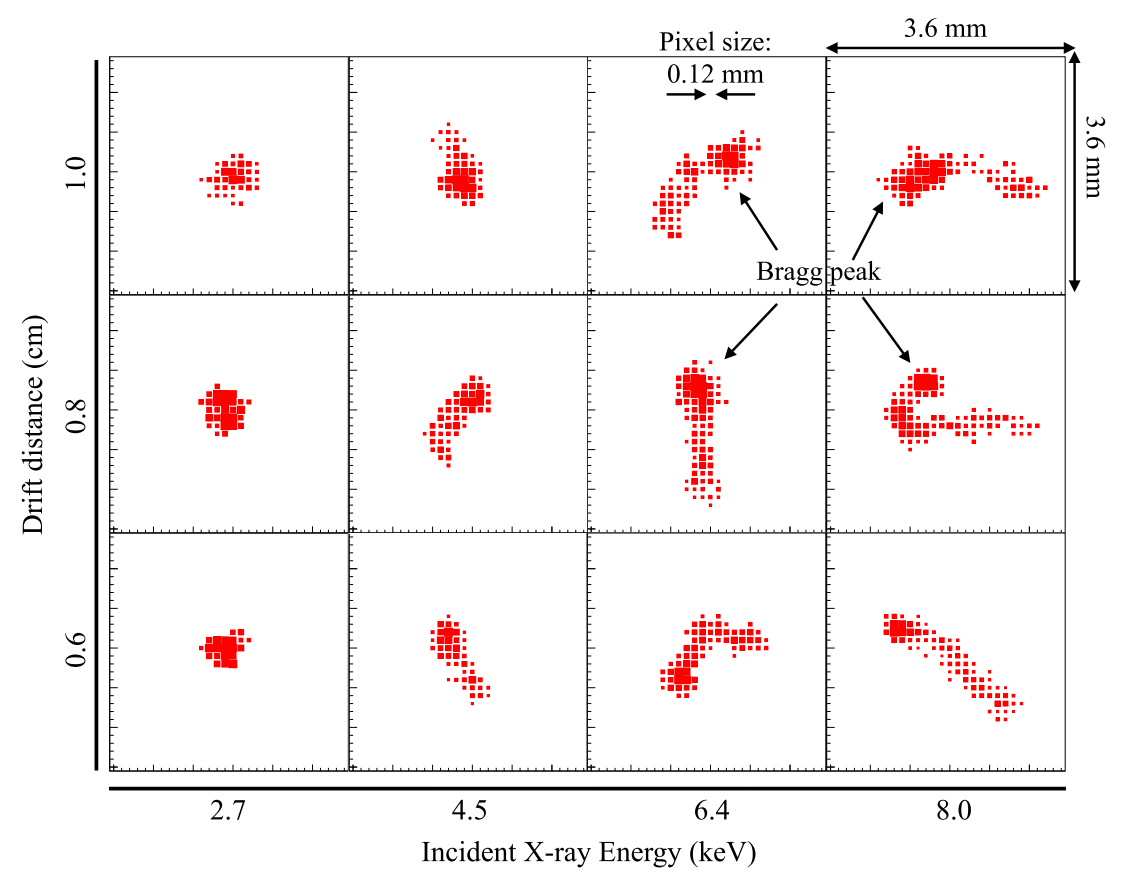

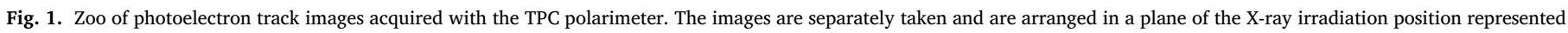

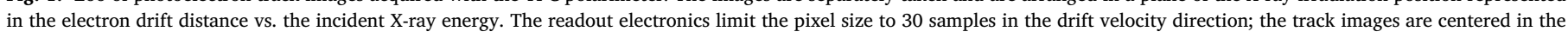

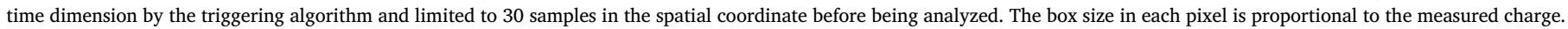

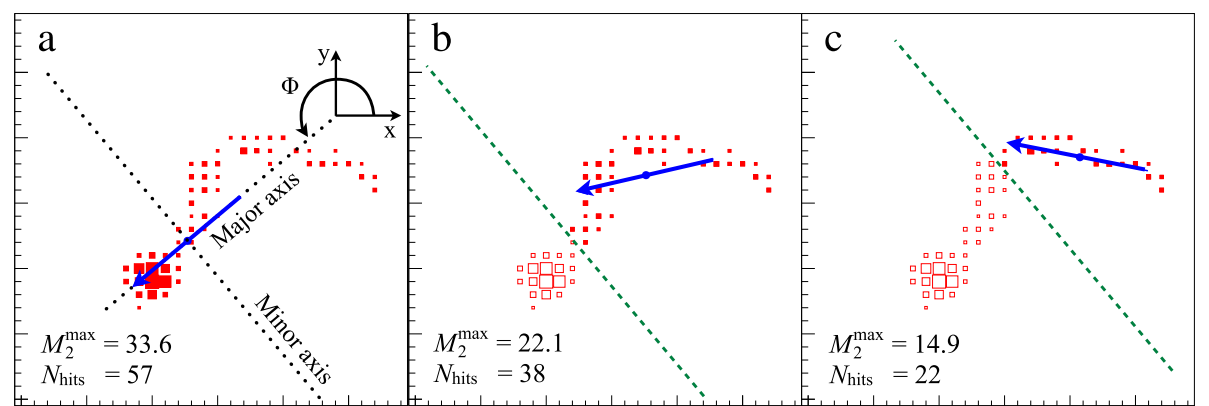

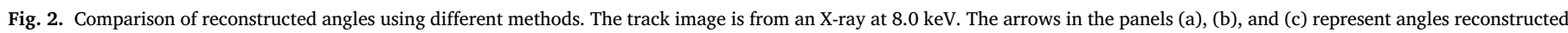

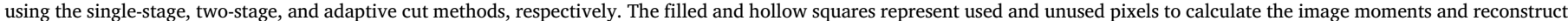

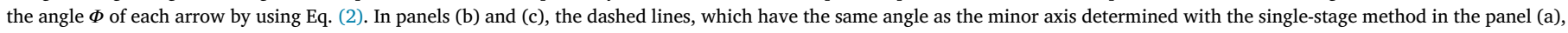
split pixels into the above two types.

which is found by scanning the two thresholds at intervals of 1 and 0.1 , respectively. The condition controls the remaining track size and avoids overcutting low-energy and round tracks for which the singlestage reconstruction without the pixel cut is the best. Fig. 2(c) shows an example of this reconstruction method named "the adaptive cut method". Although conditions with $M_{2}^{\min }$ and the third moments are individually added to the above equation, they do not improve the angular reconstruction. When the two threshold values in the condition (6) change by $10 \%$, the modulation factor varies within $1 \%$. In addition, we individually maximized the modulation factors derived from each data set taken with a monochromatic energy at a drift height in the same manner, and found that the difference of the modulation factor from that determined with the condition (6) is within $2 \%$. It shows that the method is robust against the energy and the drift height.

The angular distributions (or so-called modulation curves) determined with the three reconstruction methods described above are plotted in Fig. 3. The sinusoidal amplitude, which is proportional to the polarimetry sensitivity, is clearly improved with the revised methods. The modulation factor, $\mu$, is calculated by fitting the modulation curve to a sinusoidal model: $C\left\{\mu \cos \left[2\left(\phi-\phi_{0}\right)\right]+1\right\}$, where $C$ is a constant factor or unmodulated offset and $\phi_{0}$ is the polarization angle. In addition, the modulation factors at all the energies are corrected with the X-ray beam polarization of $94 \%$. The obtained modulation factors for $8.0 \mathrm{keV} \mathrm{X-}$ rays are $32.7 \pm 0.4,46.8 \pm 0.3$, and $56.4 \pm 0.3 \%$ determined with the single-stage, two-stage, and adaptive cut methods, respectively. The modulation factors as a function of the incident X-ray energy are shown in Fig. 4. The improved method leads to high modulation factors in the higher energy range above $5 \mathrm{keV}$. It also provides the monotonical increase of the modulation factor with the incident X-ray energy, while the single- and two-stage methods give drops to the higher energy. In the lower energy range, no drastic improvement is found because the modulation factor in that band is suppressed by track image blurring due to electron diffusion (see Fig. 1). The modulation factors determined with the adaptive cut method are $22.8 \pm 0.4,43.0 \pm 0.4,55.8 \pm 0.3$, and $56.4 \pm 0.3 \%$ at $2.7,4.5,6.4$, and $8.0 \mathrm{keV}$, respectively.

Alternatively, a novel reconstruction method based on the shortest path problem in graph theory was recently developed and applied to the GPD polarimeter [9]. The new method called "the graph-based reconstruction" in this paper is also applied to our data set. This calculation needs two parameters: the filter radius to make a reconstructed path and the distance for exponential decay to generate a distance-weighted charge map from a reconstructed interaction point. The above radius 


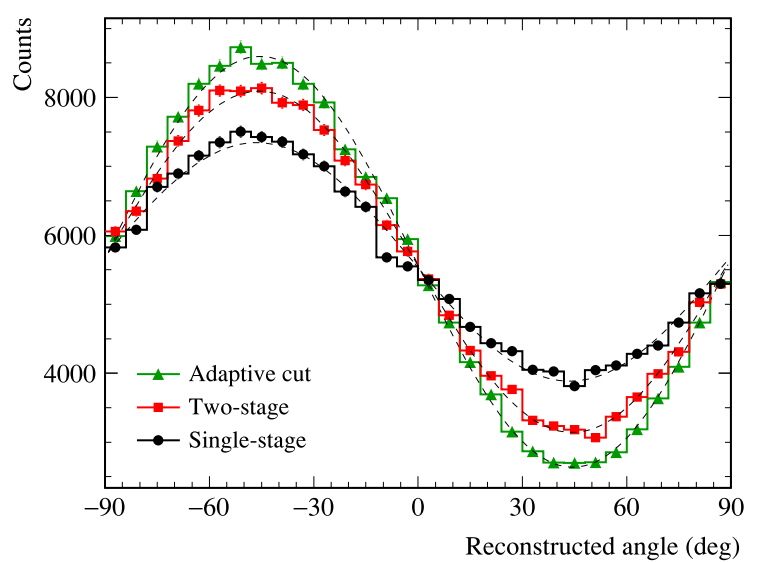

Fig. 3. Modulation curves derived from the different reconstruction methods for $8.0 \mathrm{keV}$ $\mathrm{X}$-rays. The histograms with circles, squares, and triangles are produced with the singlestage, two-stage, and adaptive cut methods, respectively. The dashed lines are the best-fit sinusoidal models to the data.

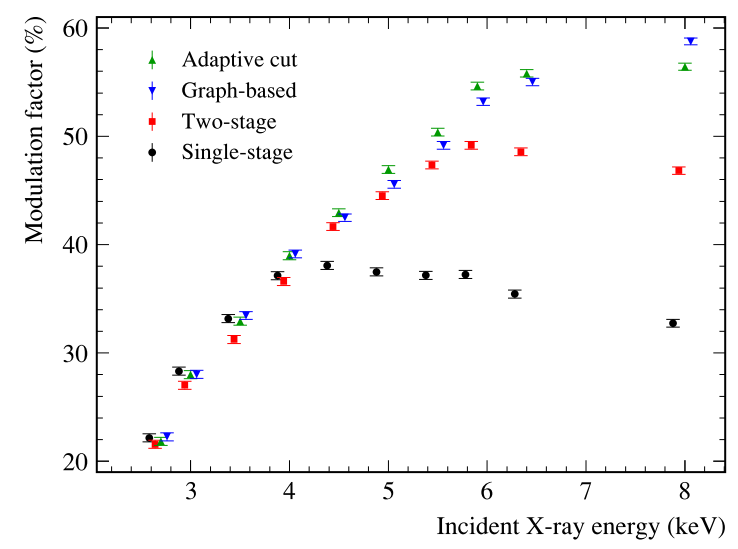

Fig. 4. Comparison of modulation factors calculated with various methods of angular reconstruction. The circles, squares, triangles, and inverted triangles show modulation factors calculated with the single-stage, two-stage, adaptive cut, and graph-based reconstruction methods, respectively. The horizontal positions of all the data points except for the triangles are slightly shifted for clarification.

and distance are 0.22 and $0.05 \mathrm{~mm}$ in Ref. [9], respectively. In our case, the two parameters are set to be 0.60 and $0.12 \mathrm{~mm}$ to maximize the modulation factor without a systematic modulation in the energy range of 2.7-10 keV. Both of them are larger than those for the GPD polarimeter because the diffusion size in the TPC polarimeter at low gas pressure is approximately double. In addition, our data set includes lower-energy and shorter tracks which require the larger radius so that the almost entire track is used to estimate the X-ray interaction point, while the GPD data set in Ref. [9] consists of 5-15 keV X-rays. The graphbased modulation factors plotted in Fig. 4 show that they are comparable to those determined with the adaptive cut method.

\section{Track selection}

Event selection with track roundness is capable of further improving polarization sensitivity. When a photoelectron is ejected in the direction nearly aligned with an incident X-ray, a 2-D photoelectron track image projected onto a plane perpendicular to the X-ray direction becomes a point or a filled circle blurred by electron diffusion. Such a track image loses the initial direction of the photoelectron or polarization information of the incident X-ray, and therefore should not be used for polarimetry analysis.
In order to quantify image roundness, two measures of eccentricity and circularity are in common use. Eccentricity, $e$, is defined here as:

$e=\sqrt{1-\frac{M_{2}^{\min }}{M_{2}^{\max }}}$.

Eccentricity has been used for track selection of X-ray gas polarimeters in the past, although it is defined in the different way (e.g. Refs. $[10,15,16])$. Alternatively, we introduce circularity, $c$, for track selection. Circularity is often defined as the ratio of the image area to square of the image perimeter. However, this value changes with image noise caused by electron diffusion and electronic noise. Instead, we use a noise-insensitive circularity measure [17] defined as:

$c=\frac{\mu_{00}^{\prime 2}}{2 \pi\left(\mu_{20}^{\prime}+\mu_{02}^{\prime}\right)}$.

This circularity measure is invariant with translations, rotations, and scaling and ranges from 0 to 1 in the same way as eccentricity. However, a circular (straight line) image has circularity of $1(0)$ and eccentricity 0 (1), showing the values mean the opposite.

Fig. 5 shows an example of photoelectron track images with eccentricity and circularity measures for $8.0 \mathrm{keV}$ X-rays. The atoll-shaped track in Fig. 5(b) has similar eccentricity to the round-shaped track in Fig. 5(a), but different circularity, showing that circularity is a better indicator of image roundness for such a curved track induced by high-energy X-rays. On the other hand, in the lower energy range where the electron diffusion size is larger than the track length, the round-shaped tracks in Fig. 6 have similar circularity due to its noiseinsensitive quality but different eccentricity. Therefore, eccentricity is more effective to select low-energy round-shaped tracks than circularity.

In order to optimize track selection with eccentricity and circularity, we define the figure of merit, $F \equiv \varepsilon \sqrt{\mu}$, where $\varepsilon$ is signal acceptance decreased by track selection and $\mu$ is the modulation factor of the polarimeter. The figure of merit is derived from the minimum detectable polarization (e.g. Refs. $[18,19]$ ) on the assumption that the background signal is negligible. In the ideal case, where $\mu$ becomes $100 \%$ by subtracting the unmodulated component from the modulation curve, $F$ can improve by $\mu^{-1 / 2}$. Since the improvement factor monotonically decreases with $\mu$ in the range from 0 to 1 , track selection can be more efficient in the lower energy range where $\mu$ is relatively low (see Fig. 4).

We search for the selection criteria which maximizes $F$. In the previous paper [10], track selection was performed only with eccentricity. In this study, selection is optimized and performed by using a rectangular region cut for tracks on a 2-D plane of eccentricity vs. circularity at each incident $\mathrm{X}$-ray energy. We empirically find that $F$ is maximized by the following condition: $\left[e>0.48\right.$ and $\left.c<c_{\mathrm{th}}(E)\right]$, where $c_{\mathrm{th}}(E)$ is the circularity threshold given by the error function, erf, of an energy,

$c_{\text {th }}(E)=-0.149 \operatorname{erf}[(E-5.53) / 1.38]+0.851$.

The energy, $E$, is obtained by scaling the measured pulse height of each event. Because $c_{\text {th }}(E)$ approaches 1 in the lower energy range, eccentricity cut is dominantly performed there.

Fig. 7 shows sensitivity improvement by using the above track selection. The modulation factors with applying track selection are $26.6 \pm 0.4,46.1 \pm 0.4,62.3 \pm 0.4$, and $61.8 \pm 0.3 \%$ at $2.7,4.5,6.4$, and $8.0 \mathrm{keV}$, respectively. Although the modulation factors are shifted up $\sim 5 \%$ at all the energies, polarimetry sensitivity, $F$, is not so effectively improved because signal acceptance decreases by $\sim 20 \%$. Compared to the previous work [10], in which we performed the adaptive method with a different loop condition and selected round tracks only with eccentricity, polarization sensitivity increases by $5 \%-10 \%$ in the higher energy range where the newly introduced selection with circularity is dominant. On the other hand, sensitivity is unchanged in the lower energy range where tracks are blurred by electron diffusion. Just for reference, the $8.0 \mathrm{keV}$ modulation factor calculated from the rejected events is $17.1 \pm 1.0 \%$, showing that the event selection is reasonable. 


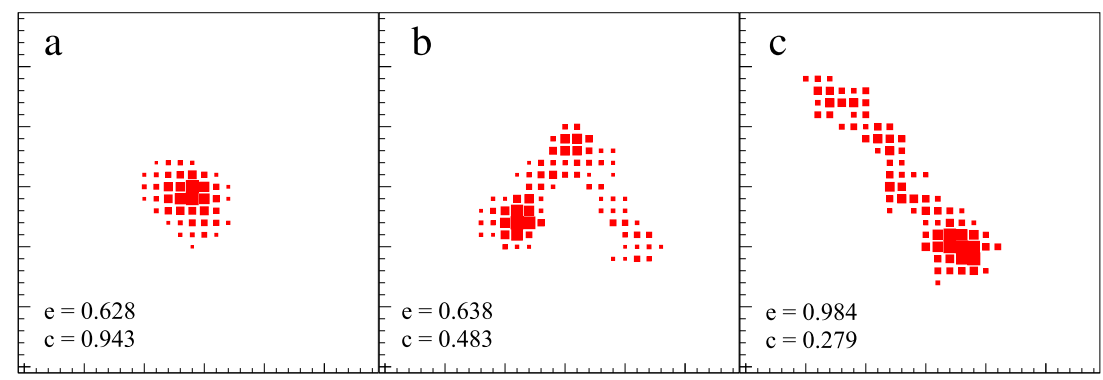

Fig. 5. Eccentricity and circularity measures for photoelectron track images produced by $8.0 \mathrm{keV}$ X-rays.

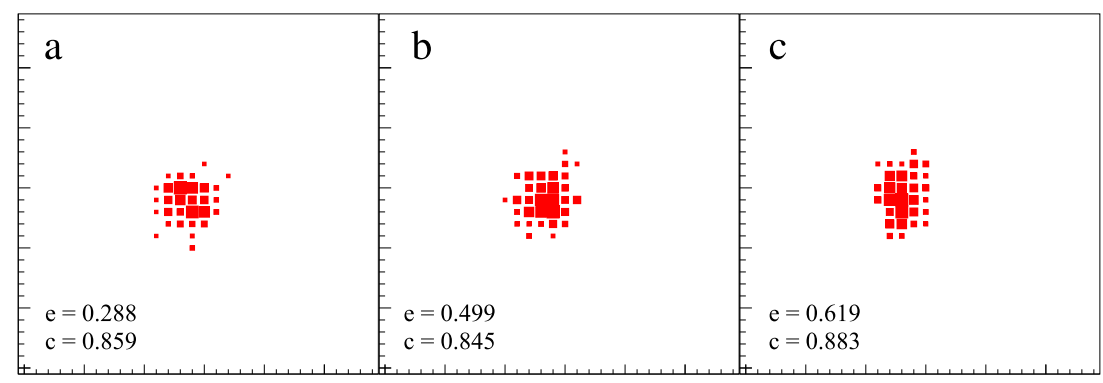

Fig. 6. Same as Fig. 5, but the incident X-ray energy is $2.7 \mathrm{keV}$.

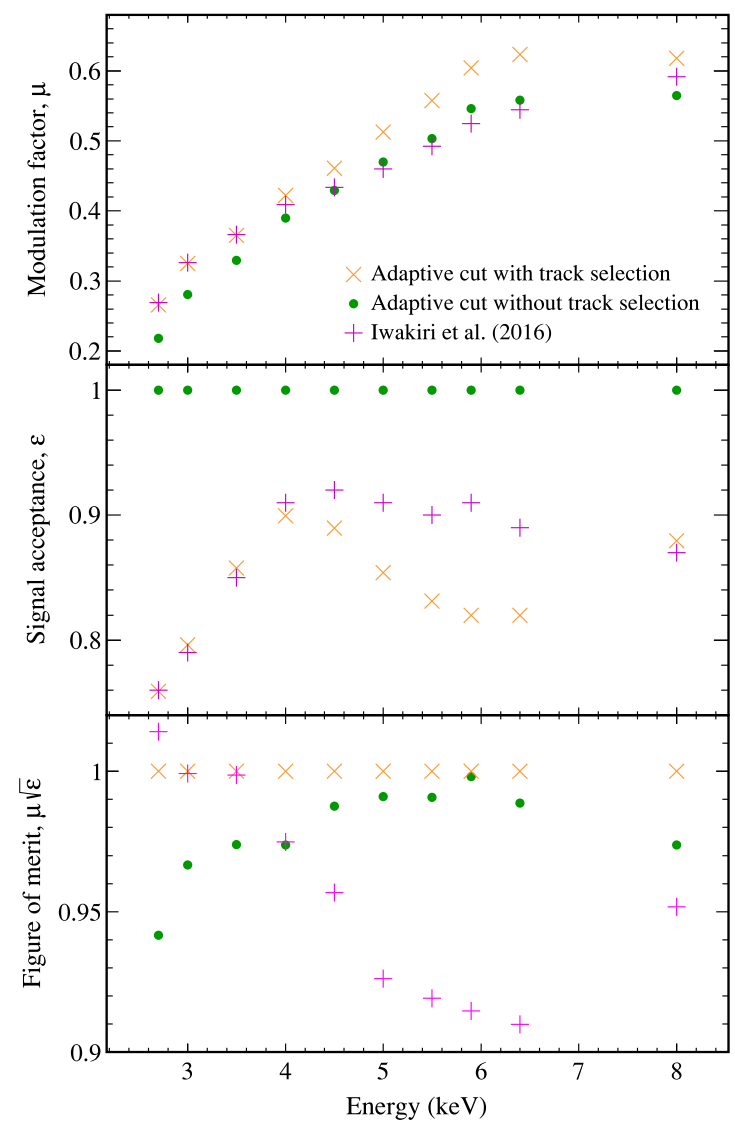

Fig. 7. Comparison of polarization sensitivity. The top, middle, and bottom panels show the modulation factor, signal acceptance, and figure of merit, respectively. The multiply and circle symbols represent values with and without the round track selection, respectively. The circle points in the top panel are the same as those in Fig. 4. The plus symbols are values in the previous work [10]. The bottom panel shows the relative improvement of polarization sensitivity with the adaptive cut method; the circle and plus symbols are figures of merit normalized by the multiply symbols at each energy.

\section{Conclusion}

We have developed angular reconstruction based on the image moments of a photoelectron track image taken with the TPC X-ray polarimeter. The algorithm processes track images in the same way with energy-independent parameters. The obtained modulation factor is $21.8 \pm 0.4 \%$ at $2.7 \mathrm{keV}$, monotonically increases with the energy, turns over around $6.4 \mathrm{keV}$, and shows a roughly constant value of $56 \%$ at the higher energy range. These modulation factors are comparable to those determined with the algorithm utilizing graph theory. Furthermore, the round track rejection method using eccentricity and the newly introduced circularity increases the modulation factors by $~ 5 \%$ at all the energies at the cost of signal loss by $\sim 20 \%$. The resulting polarization sensitivity is unchanged in the lower energy range, but is improved by $5 \%-10 \%$ above $4 \mathrm{keV}$, compared to the previous work [10].

\section{Acknowledgments}

The authors would like to acknowledge the support of Syed Khalid at the X19A beamline at the BNL-NSLS. This work was partially supported by JSPS KAKENHI Grant Number JP16H02198.

\section{References}

[1] W. Heitler, The Quantum Theory of Radiation, in: Dover Books on Physics, Dover Publications, 1954 URL https://books.google.co.jp/books?id=L7w7UpecbKYC.

[2] F. Lei, A.J. Dean, G.L. Hills, Compton polarimetry in gamma-ray astronomy, Space Sci. Rev. 82 (1997) 309-388. http://dx.doi.org/10.1023/A:1005027107614.

[3] H. Krawczynski, Analysis of the data from Compton X-ray polarimeters which measure the azimuthal and polar scattering angles, Astropart. Phys. 34 (2011) 784 788. http://dx.doi.org/10.1016/j.astropartphys.2011.02.001. arXiv:1102.1228.

[4] E. Costa, P. Soffitta, R. Bellazzini, A. Brez, N. Lumb, G. Spandre, An efficient photoelectric X-ray polarimeter for the study of black holes and neutron stars, Nature 411 (2001) 662-665. arXiv:astro-ph/0107486.

[5] R. Bellazzini, G. Spandre, M. Minuti, L. Baldini, A. Brez, L. Latronico, N. Omodei, M. Razzano, M.M. Massai, M. Pesce-Rollins, C. Sgró, E. Costa, P. Soffitta, H. Sipila, E. Lempinen, A sealed Gas Pixel Detector for X-ray astronomy, Nucl. Instrum. Methods Phys. Res. A 579 (2007) 853-858. http://dx.doi.org/10.1016/j.nima.2007.05.304. arXiv:astro-ph/0611512.

[6] J.K. Black, R.G. Baker, P. Deines-Jones, J.E. Hill, K. Jahoda, X-ray polarimetry with a micropattern TPC, Nucl. Instrum. Methods Phys. Res. A 581 (2007) 755-760. http://dx.doi.org/10.1016/j.nima.2007.08.144. 
[7] J.E. Hill, J.K. Black, T.J. Emmett, T. Enoto, K.M. Jahoda, P. Kaaret, D.S. Nolan, T. Tamagawa, Design improvements and x-ray performance of a time projection chamber polarimeter for persistent astronomical sources, in: Society of PhotoOptical Instrumentation Engineers (SPIE) Conference Series, Vol. 9144 of Society of Photo-Optical Instrumentation Engineers (SPIE) Conference Series, 2014, p. 1, http://dx.doi.org/10.1117/12.2057259.

[8] R. Bellazzini, F. Angelini, L. Baldini, A. Brez, E. Costa, G. Di Persio, L. Latronico, M.M. Massai, N. Omodei, L. Pacciani, P. Soffitta, G. Spandre, Novel gaseus Xray polarimeter: data analysis and simulation, in: S. Fineschi (Ed.), Polarimetry in Astronomy, in: Proc. SPIE, vol. 4843, 2003, pp. 383-393. http://dx.doi.org/10. $1117 / 12.459381$.

[9] T. Li, M. Zeng, H. Feng, J. Cang, H. Li, H. Zhang, Z. Zeng, J. Cheng, H. Ma, Y. Liu, Electron track reconstruction and improved modulation for photoelectric Xray polarimetry, Nucl. Instrum. Methods Phys. Res. A 858 (2017) 62-68. http://dx. doi.org/10.1016/j.nima.2017.03.050. URL http://www.sciencedirect.com/science/ article/pii/S0168900217304035.

[10] W.B. Iwakiri, J.K. Black, R. Cole, T. Enoto, A. Hayato, J.E. Hill, K. Jahoda, P. Kaaret, T. Kitaguchi, M. Kubota, H. Marlowe, R. McCurdy, Y. Takeuchi, T. Tamagawa, Performance of the praxys x-ray polarimeter, Nucl. Instrum. Methods Phys. Res. A 838 (2016) 89-95. http://dx.doi.org/10.1016/j.nima.2016.09.024. arXiv:1610. 06677.

[11] T. Enoto, J.K. Black, T. Kitaguchi, A. Hayato, J.E. Hill, K. Jahoda, T. Tamagawa, K. Kaneko, Y. Takeuchi, A. Yoshikawa, H. Marlowe, S. Griffiths, P.E. Kaaret, D. Kenward, S. Khalid, Performance verification of the Gravity and Extreme Magnetism Small explorer (GEMS) x-ray polarimeter, in: Society of Photo-Optical Instrumentation Engineers (SPIE) Conference Series, Vol. 9144 of Society of Photo-Optical Instrumentation Engineers (SPIE) Conference Series, 2014, p. 4, http://dx.doi.org/ $10.1117 / 12.2056841$.
[12] T. Tabata, P. Andreo, K. Shinoda, An analytic formula for the extrapolated range of electrons in condensed materials, Nucl. Instrum. Methods Phys. Res. 119 (1996) 463-470. http://dx.doi.org/10.1016/S0168-583X(96)00687-8.

[13] S.F. Biagi, Monte Carlo simulation of electron drift and diffusion in counting gases under the influence of electric and magnetic fields, Nucl. Instrum. Methods Phys. Res. A 421 (1999) 234-240. http://dx.doi.org/10.1016/S0168-9002(98)01233-9.

[14] K. Castleman, Digital Image Processing, in: Prentice-Hall signal processing series, Prentice Hall, 1996 URL https://books.google.co.jp/books?id= OYUYAQAAIAAJ.

[15] F. Muleri, P. Soffitta, L. Baldini, R. Bellazzini, A. Brez, E. Costa, S. Fabiani, F. Krummenacher, L. Latronico, F. Lazzarotto, M. Minuti, M. Pinchera, A. Rubini, C. Sgró, G. Spandre, Spectral and polarimetric characterization of the Gas Pixel Detector filled with dimethyl ether, Nucl. Instrum. Methods Phys. Res. A 620 (2010) 285-293. http://dx.doi.org/10.1016/j.nima.2010.03.006. arXiv:1003.6009.

[16] W.H. Baumgartner, T. Strohmayer, T. Kallman, J.K. Black, J.E. Hill, J.H. Swank, K.M. Jahoda, GEMS x-ray polarimeter performance simulations, in: Space Telescopes and Instrumentation 2012: Ultraviolet To Gamma Ray, in: Proc. SPIE, vol. 8443, 2012, p. 84434K. http://dx.doi.org/10.1117/12.927270.

[17] J. Žunić, K. Hirota, P.L. Rosin, A hu moment invariant as a shape circularity measure, Pattern Recognit. 43 (1) (2010) 47-57. http://dx.doi.org/10. 1016/j.patcog.2009.06.017. URL http://www.sciencedirect.com/science/article/ pii/S0031320309002660.

[18] M.C. Weisskopf, R.F. Elsner, S.L. O'Dell, On understanding the figures of merit for detection and measurement of x-ray polarization, in: Space Telescopes and Instrumentation 2010: Ultraviolet To Gamma Ray, in: Proc. SPIE, vol. 7732, 2010, p. 77320E. http://dx.doi.org/10.1117/12.857357. arXiv:1006.3711.

[19] T.E. Strohmayer, T.R. Kallman, On the statistical analysis of x-ray polarization measurements, Appl. J. 773 (2013) 103. http://dx.doi.org/10.1088/0004-637X/ 773/2/103. arXiv:1306.3885. 